\title{
Understanding Gender Stereotypes and Electoral Success from Visual Self-presentations of Politicians in Social Media
}

\author{
Danni Chen \\ UCLA \\ dannichen236@gmail.com
}

\author{
Kunwoo Park \\ UCLA \\ kunwpark@ucla.edu
}

\author{
Jungseock Joo \\ UCLA \\ jjoo@comm.ucla.edu
}

\begin{abstract}
Social media have been widely used as a platform for political communication, promoting firsthand dialogue between politicians and the public. This paper studies the role of visual self-presentation in social media in political campaigns with a primary focus on gender stereotypical cues exhibited in Facebook timeline posts of 562 candidates in the 2018 U.S. general elections. We train a convolutional neural network (CNN) that infers gender stereotypes from the photographs based on crowdsourced annotations. Using regression analysis, we find that masculine traits are predictive factors for winning elections for both gender and parties. In contrast, feminine traits are not correlated with electoral success. Prediction experiments show that the visual traits on gender stereotypes can predict the election outcomes with an accuracy of 0.739 , which was better than the performance (0.724) of making a direct prediction from the raw photographs. Our study demonstrates that the automated visual content analysis can reliably measure subtle, emotional, and subjective personal trait dimensions from political images, thereby enabling systematic investigations on multi-modal political communication via social media.
\end{abstract}

\section{CCS CONCEPTS}

- Information systems $\rightarrow$ Social networks; $\bullet$ Human-centered computing $\rightarrow$ Collaborative and social computing; • Computing methodologies $\rightarrow$ Computer vision; $\bullet$ Applied computing $\rightarrow$ Law, social and behavioral sciences.

\section{KEYWORDS}

Social Media based Election Prediction; Visual Communication; Gender Stereotypes; Computer Vision

\section{ACM Reference Format:}

Danni Chen, Kunwoo Park, and Jungseock Joo. 2020. Understanding Gender Stereotypes and Electoral Success from Visual Self-presentations of Politicians in Social Media. In foint Workshop on Aesthetic and Technical Quality Assessment of Multimedia and Media Analytics for Societal Trends (ATQAM/MAST'20), October 12-16, 2020, Seattle, WA, USA. ACM, New York, NY, USA, 5 pages. https://doi.org/10.1145/3423268.3423583

Permission to make digital or hard copies of part or all of this work for personal or classroom use is granted without fee provided that copies are not made or distributed for profit or commercial advantage and that copies bear this notice and the full citation on the first page. Copyrights for third-party components of this work must be honored For all other uses, contact the owner/author(s).

ATQAM/MAST'20, October 12-16, 2020, Seattle, WA, USA

(c) 2020 Copyright held by the owner/author(s).

ACM ISBN 978-1-4503-8154-3/20/10.

https://doi.org/10.1145/3423268.3423583

\section{INTRODUCTION}

In the past decade, social media have become a powerful platform for political discourses, civic engagement, and electoral campaigning $[5,12]$. The new technology has helped achieve decentralized and personalized public communication by enabling selfpresentation and direct exchanges of ideas between politicians and citizens $[7,23,24]$, unlike the mass media where professional journalists act as gatekeepers who decide what and how politicians are presented [28]. More citizens are attending social media as their primary news sources, and consequently, politicians are also quickly shifting to social media to reach out to their supporters.

A considerable body of literature has examined the effects of gender stereotypical representations of politicians in media on voter preference and electoral success. Many studies have reported that compared to male politicians, female politicians are often discussed in terms of their role as mothers on news coverage and, therefore, possibly lose viability as competent candidates [4]. In social media, however, the gender stereotype may be alleviated because candidates themselves can choose what to post on their accounts. This implies that social media may be an effective platform for the self-presentation of politicians underrepresented or portrayed stereotypically in the mass media.

The main objective of our paper is to understand how politicians attempt to establish themselves as competent leaders on social media. In particular, we mainly investigate the role of the visual dimension of political messages, i.e., photographs that politicians post on their accounts. Previous studies have explored the effects of candidates' visual appearance on voter perception in TV, print media, and social media [10,21]. Nevertheless, it has rarely been attempted to analyze a large amount of visual data due to the difficulty in processing large-scale visual data. We use a computer vision approach to automatically analyze visual content on the dimensions related to gender stereotypes, from which we examine the role of visual self-presentation of candidates in election campaigning. Incorporated with image and text categories identified from the politicians' Facebook posts, we will discuss the importance of visual gender stereotypes through regression analysis and prediction experiments.

\section{RELATED WORK}

Social media and election. Many scholars have actively studied the role of social media in political communication and campaigning. In particular, these platforms provide a substantial amount of visual data on political campaigns, making the dataset readily available for quantitative analysis [3, 9, 19, 27, 29, 31]. The following section will discuss how some key traits have been identified from the existing literature and are incorporated into our study. 
Visual presentation of politicians and gender stereotypes. A large volume of research has examined the gender stereotypes of both male and female politicians and their effects on public perception and candidate evaluation. It is generally accepted that voters interpret masculinity as a sign of competency and may penalize female politicians who lack typical masculine features [15, 20,26]. For example, a study reported that female candidates benefited from presenting themselves explicitly as caregivers [2]. On the other hand, a backlash effect was also reported for Democratic female candidates expressing masculine visuals, whereas Republican female candidates are not disadvantaged by such incongruities due to Republican Party stereotypes [2].

Automated visual content analysis. Computational social scientists have recently used computer vision and deep learning for the large-scale visual content analysis of massive data from media. Such methods can significantly improve the efficiency of coding and provide new insights on human behaviors and social events such as election [18, 32, 33, 36, 38], collective actions and protest [34, 37], and political traits and ideology inference $[14,17,35]$. Furthermore, since a substantial portion of online communication is in the form of visual data, image data offers unprecedented potential for social science research on the web and media [30].

\section{DATA AND METHODOLOGY}

\subsection{Data Collection}

Using Facebook Graph API and selenium, we collected a novel Facebook dataset of 800 politicians who ran for the 2018 U.S. House, Senate, or Governor election. The candidates and the election outcomes (voting shares) were obtained from Ballotpedia, and their Facebook accounts were manually confirmed. We collected photographs, captions, number of comments, and posting time. Along with Facebook photographs, posting time was examined to filter the photographs by the year 2018 (January 1, 2018 - November 5, 2018). Facebook was chosen as our target domain of analysis because it has been widely used as a platform for political discussions in recent elections in the U.S.

Some images display only large text or big logos without much photographic content without depicting the visual traits of candidates. We excluded these images by training a classifier from a small annotation set we created. Among the politicians who had a Facebook account and posted at least five images, we only considered the complete pairs for this study's analyses. In other words, politicians without opponents in this dataset are discarded. The final dataset used for analysis is composed of 80,131 images shared by 562 politicians, which is well balanced across gender and party.

\subsection{Data Annotation on Personal Traits}

Personal traits are a critical basis to understand how voters perceive candidates and predict their competence. However, quantifying the personal traits can be challenging because there exist several potential trait dimensions one can consider. This study aims to provide a method that automatically quantifies scores for the selected traits by training a deep neural network with crowdsourced annotations. To the end, we first construct a list of traits by borrowing from research on personal traits of politicians [1, 6, 11, 17, 18, 22, 25]. Along with Masculinity and Femininity, we target the following
22 trait dimensions that could affect perception of electorates and election outcomes: Ordinary, Elitist, Qualified, Ambitious, Attractive, Threatening, Communal, Formal, Patriotic, Professional, Energetic, Trustworthy, Agreeable, Reassuring, Friendly, Caring, Maternal, Credible, Competent and Confident.

We employed Amazon Mechanical Turk to collect human judgment ratings on each of the 22 trait dimensions. Note that the aim of the annotation task was not to identify the truth but to collect subjective opinions of people on the politicians' personal traits; the perceived traits indeed affect the voting decision of electorates. We randomly sampled at least five images per candidate and collected 8,954 images while ensuring the balance between gender and political affiliation. We assigned ten annotators in the U.S. to each image and ask to rate to which extent the given image exhibits each trait on a five-point scale (1 to 5 ). The results were aggregated by averaging the responses to each image. We excluded ratings from the annotators who indicated that they recognize the politicians in the given photographs as their evaluations might be biased.

As we ask for subjective opinions, instead of conventional measures such as Cronbach's alpha, we adopted a method suggested in a study in a similar setting [16]. In particular, the method split the annotations into two groups and then evaluate the correlation of average scores between the two via Pearson's $r$. This measure intuitively tells us how well the first half of the annotations predict the ratings made by the second half of the annotators. The results show that the crowdsourced trait scores achieved an acceptable level of inter-annotator agreements for each trait dimension $(>.2)$, which suggests the reliability of the annotations.

Table 1: Prediction accuracy for the personal traits measured on the test set by Pearson's Correlation.

\begin{tabular}{lr|lr} 
Trait & Accuracy & Trait & Accuracy \\
\hline Feminine & 0.870 & Trustworthy & 0.819 \\
Formal & 0.867 & Energetic & 0.816 \\
Professional & 0.859 & Maternal & 0.810 \\
Agreeable & 0.853 & Masculine & 0.802 \\
Reassuring & 0.853 & Communal & 0.797 \\
Friendly & 0.851 & Ambitious & 0.797 \\
Caring & 0.852 & Patriotic & 0.797 \\
Credible & 0.844 & Attractive & 0.752 \\
Competent & 0.838 & Elitist & 0.650 \\
Qualified & 0.827 & Ordinary & 0.610 \\
Confident & 0.823 & Threatening & 0.339 \\
\hline
\end{tabular}

\subsection{Automatic Trait Inference from Images}

We trained a CNN model to quantify the 22 trait values from each photograph automatically. In particular, we employed ResNet34 [13]. From the model pretrained on the ImageNet data, we replaced the last layer with the 22-dimensional output and added a sigmoid layer to ensure the computed personal traits ranging from 0 to 1 . We fine-tuned the network by minimizing the sum of the 22 different mean square errors. During training, we employed the Stochastic Gradient Descent (SGD) optimizer with a learning rate of 0.03 and a momentum value of 0.9 . Out of 8,954 labeled images, 500 were randomly selected for testing the trait inference model. 
High Masculinity

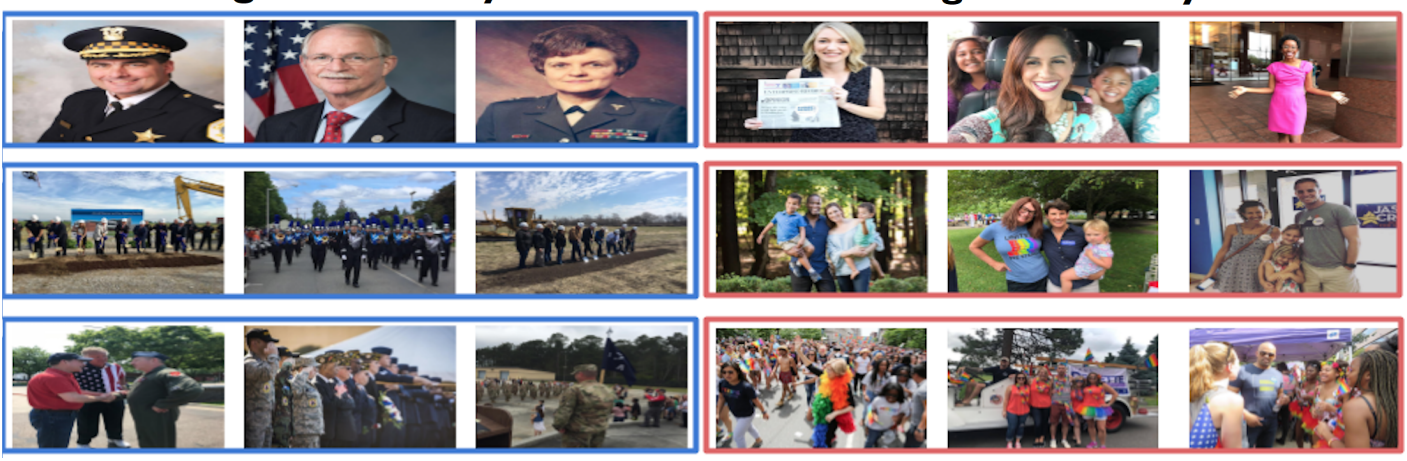

Figure 1: Examples of image clusters of high Masculine(left) and high Feminine scores (right) identified by k-means $(k=300)$.

Table 1 presents the accuracy of our trait inference model, measured on a separate test set with the average rating per image. The CNN model trained on the annotated scores could predict the trait values with high accuracy scores similar to or higher than 0.8 , except for Attractive, Elitist, Ordinary, and Threatening. Considering the low prediction accuracy, we did not utilize the four dimensions in the following analyses.

\section{RESULTS}

\subsection{Understanding Visual Gender Stereotypes}

An essential question in our study is to understand what constitutes femininity and masculinity. To illustrate how they are displayed differently, we conducted a clustering analysis using the trait prediction model. In particular, we utilized the vector of the penultimate layer by feeding each image into the trait model as input. Then we took the top 10,000 images with the highest femininity score and the top 10,000 with the highest masculinity score and clustered them using the $k$-means algorithm with $k=300$. Figure 1 displays samples of the image clusters with a high masculinity and femininity score, respectively. Images from the same cluster are bounded by a border. In the figure, we can clearly see that femininity (red) is formed around the candidates and highlights their friendly, attractive, and maternal personality along with visual cues such as smile and family whereas masculinity (blue) is conveyed through strong and authoritative activities, such as military, handshaking and construction sites.

To better understand the context of the politicians' posts on social media and how they are associated with gender stereotypes (and in turn election outcomes), we further analyzed topics that appear in image and text.

Using the Google Vision API, we investigated the top-10 concepts in images of high masculinity and high femininity by calculating the expected frequency for each gender based on the frequency of images shared by each gender; then, we computed the chi-square value based on the difference from the actual frequency and the expected. Results show that male-dominated occupations (Military, Engineering) appeared more frequently in images shared by the male politicians; on the contrary, emotion-related concepts (Friendship, Happy, Laugh, Hug) were highly associated with images shared by the female politicians. Note that the identified image categories also appeared in the masculine and feminine clusters in Figure 1: for example, images of politicians in a military event or those doing handshakes are observed in the masculine clusters while politicians with smiling or laughing faces appeared in the feminine clusters. The two different analyses' common trend suggests that masculinity and femininity could be visually represented by certain image concepts, such as context and type of persons.

Similarly, we analyzed texts using Empath [8]. Similar to Google Vision API, the library returns the list of text categories for a given text. We fed the caption of a Facebook post as the input of Empath. In this analysis, we found that while work- and achievement-related text categories were prominently used by the male politicians (Office, Achievement), the female politicians tend to use emotion- and family-related text (Affection, Children). In addition to the image analysis, the text analysis suggests that male and female politicians cover different topics, and hence masculinity and femininity may be captured by distinct concepts.

\subsection{Identifying Important Traits for Election}

To investigate the predictive roles of each of the stereotype dimensions with ruling out the effects of the other factors, we utilized two regression models for predicting voting shares (continuous) and whether a given politician win the election (binary). In particular, ordinary least square (OLS) and logistic regression were fitted using the three categories of features: the political traits estimated by CNN (Personal trait), the image categories identified via Google Vision API (Image category), and the text categories found by Empath (Text category). To reduce the number of independent variables in the regression models, we first fitted a model for each set of features (e.g., Personal trait) and only considered the significant variables for the model using all the categories. Dummy variables on gender $($ Female $=1)$ and party (Democrats $=1$ ) were included as control variables; therefore, the predictive power of significant variables from each model is also independent of the effects of gender and party.

Table 2 presents the regression results. Here we make three sets of observations. First, the models can predict the election outcomes with the Adjusted R-squared of 0.282 and the Pseudo R-squared of 0.2726; that is, how the politicians present themselves on social media, including the gender stereotypical traits, were important for explaining election outcomes. Second, among the personal traits 
Table 2: Fitted regression results. Bold text indicates statistically significant variables.

(a) OLS regression for voting shares

\begin{tabular}{clrrr}
\hline Category & \multicolumn{1}{c}{ Feature } & Coefficient & Std. Error & P-value \\
\hline & (Intercept) & -0.2329 & 0.128 & 0.069 \\
\hline \multirow{2}{*}{ Control } & Gender & 0.0079 & 0.017 & 0.639 \\
& Party & 0.0339 & 0.012 & 0.004 \\
\hline \multirow{3}{*}{ Personal } & Masculine & 0.7675 & 0.346 & 0.027 \\
trait & Feminine & 0.3801 & 0.316 & 0.229 \\
& Professional & 0.2096 & 0.243 & 0.388 \\
& Patriotic & 0.0481 & 0.229 & 0.834 \\
& Energetic & 0.1174 & 0.378 & 0.757 \\
\hline \multirow{4}{*}{ Image } & T-shirt & -0.3655 & 0.103 & $<0.001$ \\
category & Smile & -0.2548 & 0.076 & 0.001 \\
& Vehicle & -0.1122 & 0.084 & 0.183 \\
& Employment & 0.1369 & 0.082 & 0.098 \\
& Social group & 0.2729 & 0.088 & 0.005 \\
& & & & \\
\hline \multirow{2}{*}{ Text } & Tourism & 3.7182 & 1.606 & 0.021 \\
category & Journalism & -5.8585 & 2.281 & 0.010 \\
& Business & 2.0814 & 0.776 & 0.008 \\
& Achievement & 1.6222 & 0.800 & 0.043 \\
\hline \multirow{2}{*}{ Adjusted R-squared } & & 0.282 \\
\hline
\end{tabular}

estimated by $\mathrm{CNN}$, masculinity was the only significant feature for predicting election outcomes. This finding is congruent with the finding of the previous studies which discusses the importance of masculinity through manual analysis [20,26]. Third, there were significant variables from image and text categories identified from the Facebook posts. Among the Google Vision categories identified from the photographs, both regression models exhibited a statistical significance on T-shirt and Smile, which was more used in the images of female politicians. By contrast, among the text categories of Empath, Business and Achievement were the significant variables with a positive association with the election outcome. Those two text categories were more frequently used by male politicians. Together, the image and text categories captured from politician's Facebook posts support the advantage of exploiting masculine traits for winning the election.

\subsection{Election Outcome Prediction from Images}

The last step of the analysis was to test the predictive power of visual self-presentation for the election outcome. To this end, we mimic the election setting using 5 -fold cross-validation. In particular, we split the list of positions (e.g., Governor in California) into five, and therefore it ensures every politician having the opponent in the same fold. We decided a winner when a politician achieves a higher score than its counterpart.

In particular, we utilized the two different models to evaluate the relative importance of the stereotyped traits for predicting election outcomes: (i) Direct model and (ii) Trait-based model. The direct model takes an image as input and returns a probability of winning the election. We fine-tuned ResNet-34 pretrained on ImageNet by replacing the last layer with a sigmoid layer. We used up to 300 images for each politician for training to prevent overfitting. For inference, we took the average of the model outputs of all images a candidate posted. The trait-based model is a logistic regression (b) Logistic regression for win/lose

\begin{tabular}{clrrr}
\hline Category & \multicolumn{1}{c}{ Feature } & Coefficient & Std. Error & P-value \\
\hline & (Intercept) & -7.8704 & 3.063 & 0.010 \\
\hline \multirow{2}{*}{ Control } & Gender & 0.2393 & 0.361 & 0.507 \\
& Party & 0.1997 & 0.254 & 0.432 \\
\hline \multirow{3}{*}{ Personal } & Masculine & 23.6045 & 8.056 & 0.003 \\
trait & Feminine & 12.5612 & 7.453 & 0.092 \\
& Professional & -4.4074 & 5.588 & 0.430 \\
& Patriotic & 3.2534 & 5.493 & 0.554 \\
& Energetic & -12.7146 & 9.201 & 0.167 \\
\hline \multirow{4}{*}{ Image } & T-shirt & -6.8475 & 2.595 & 0.008 \\
category & Smile & -4.7920 & 1.989 & 0.016 \\
& Vehicle & -5.1648 & 1.821 & 0.005 \\
& Employment & 4.3472 & 1.914 & 0.023 \\
& Social group & 4.7296 & 3.029 & 0.118 \\
& Team & 1.6945 & 1.733 & 0.328 \\
\hline \multirow{2}{*}{ Text } & Home & -49.9361 & 26.695 & 0.061 \\
category & Military & 84.8846 & 43.551 & 0.051 \\
& Business & 51.2308 & 18.325 & 0.005 \\
& Achievement & 41.5964 & 17.431 & 0.017 \\
\hline \multirow{2}{*}{ Pseudo R-squared } & & 0.2726 \\
\hline & & & &
\end{tabular}

classifier that predicts whether a candidate wins from the inferred visual traits for the politician. To have a vector for each politician, we took an average of each of the trait dimensions for all images shared by the politician.

From the evaluation experiments, we made two key findings. First, the image-based direct model achieved a fair accuracy of 0.724 with a significance level of 0.001 by McNemar's test. This performance suggests that the visual self-presentation of politicians contains predictive cues for election outcomes. Second, the traitbased model achieved an accuracy of 0.739 with a significance level of 0.001 , which was slightly better than the image-based direct model's performance. This suggests the gender-stereotyped visual traits are essential features for classifying winners and losers, as we also identified masculinity as a significant factor in Table 2.

\section{CONCLUSION}

In this paper, we investigated the role of visual self-presentation of political candidates in social media in campaigning using 80,131 photographs shared by the 562 political candidates in the 2018 U.S. general election. While the previous research in political science has discussed the importance of visual information [10], the main challenge in the visual data analysis in political science was on how to conduct a systematic analysis on a large-scale data. This study employed an advanced computer vision method that allows an automated prediction of political traits displayed in photographs to fill the gap. For computer scientists, we believe this study also provides a promising view for future studies investigating gender stereotypes and bias through online multimedia.

\section{ACKNOWLEDGMENTS}

This work was supported by NSF SBE-SMA \#1831848 and Hellman Fellowship. 


\section{REFERENCES}

[1] Nichole M Bauer. 2017. The effects of counterstereotypic gender strategies on candidate evaluations. Political Psychology 38, 2 (2017), 279-295.

[2] Nichole M Bauer and Colleen Carpinella. 2018. Visual information and candidate evaluations: the influence of feminine and masculine images on support for female candidates. Political Research Ouarterly 71, 2 (2018), 395-407.

[3] Adam Bermingham and Alan Smeaton. 2011. On using Twitter to monitor political sentiment and predict election results. In Proceedings of the Workshop on Sentiment Analysis where AI meets Psychology (SAAIP 2011). 2-10.

[4] Dianne G Bystrom, Terry A Robertson, and Mary Christine Banwart. 2001. Fram ing the fight: An analysis of media coverage of female and male candidates in primary races for governor and US Senate in 2000. American Behavioral Scientist 44, 12 (2001), 1999-2013.

[5] Danish Contractor and Tanveer Afzal Faruquie. 2013. Understanding election candidate approval ratings using social media data. In Proceedings of the 22nd International Conference on World Wide Web. ACM, 189-190.

[6] Tessa M Ditonto, Allison J Hamilton, and David P Redlawsk. 2014. Gender stereotypes, information search, and voting behavior in political campaigns. Political Behavior 36, 2 (2014), 335-358.

[7] Gunn Sara Enli and Eli Skogerbø. 2013. Personalized campaigns in party-centred politics: Twitter and Facebook as arenas for political communication. Information, communication \& society 16, 5 (2013), 757-774.

[8] Ethan Fast, Binbin Chen, and Michael S Bernstein. 2016. Empath: Understanding topic signals in large-scale text. In Proceedings of the 2016 CHI Conference on Human Factors in Computing Systems. 4647-4657.

[9] Daniel Gayo-Avello, Panagiotis Takis Metaxas, and Eni Mustafaraj. 2011. Limits of electoral predictions using twitter. In Fifth International AAAI Conference on Weblogs and Social Media.

[10] Maria Elizabeth Grabe and Erik Page Bucy. 2009. Image bite politics: News and the visual framing of elections. Oxford University Press.

[11] Maria Elizabeth Grabe and Erik P Bucy. 2010. Image bite analysis of political visuals. Sourcebook for political communication research: Methods, measures, and analytical techniques (2010), 209-237.

[12] Todd Graham, Marcel Broersma, Karin Hazelhoff, and Guido Van'T Haar. 2013 Between broadcasting political messages and interacting with voters: The use of Twitter during the $2010 \mathrm{UK}$ general election campaign. Information, communication \& society 16, 5 (2013), 692-716.

[13] Kaiming He, Xiangyu Zhang, Shaoqing Ren, and Jian Sun. 2016. Deep residual learning for image recognition. In Proceedings of the IEEE conference on computer vision and pattern recognition. $770-778$.

[14] Xinyue Huang and Adriana Kovashka. 2016. Inferring visual persuasion via body language, setting, and deep features. In Proceedings of the IEEE Conference on Computer Vision and Pattern Recognition Workshops. 73-79.

[15] Leonie Huddy and Nayda Terkildsen. 1993. The consequences of gender stereotypes for women candidates at different levels and types of office. Political Research Quarterly 46, 3 (1993), 503-525.

[16] Phillip Isola, Jianxiong Xiao, Antonio Torralba, and Aude Oliva. 2011. What makes an image memorable? In CVPR. IEEE, 145-152.

[17] Jungseock Joo, Weixin Li, Francis F Steen, and Song-Chun Zhu. 2014. Visual persuasion: Inferring communicative intents of images. In Proceedings of the IEEE conference on computer vision and pattern recognition. 216-223.

[18] Jungseock Joo, Francis F Steen, and Song-Chun Zhu. 2015. Automated facial trait judgment and election outcome prediction: Social dimensions of face. In Proceedings of the IEEE international conference on computer vision. 3712-3720.

[19] Andreas Jungherr, Pascal Jürgens, and Harald Schoen. 2012. Why the pirate party won the german election of 2009 or the trouble with predictions: A response to tumasjan, a., sprenger, to, sander, pg, \& welpe, im "predicting elections with twitter: What 140 characters reveal about political sentiment". Social science computer review 30, 2 (2012), 229-234.

[20] Anne M Koenig, Alice H Eagly, Abigail A Mitchell, and Tiina Ristikari. 2011. Are leader stereotypes masculine? A meta-analysis of three research paradigms. Psychological bulletin 137, 4 (2011), 616.

[21] Jamie Mahoney, Tom Feltwell, Obinna Ajuruchi, and Shaun Lawson. 2016. Constructing the visual online political self: an analysis of Instagram Use by the Scottish Electorate. In Proceedings of the 2016 CHI Conference on Human Factors in Computing Systems. ACM, 3339-3351.

[22] Kyle Mattes, Michael Spezio, Hackjin Kim, Alexander Todorov, Ralph Adolphs, and R Michael Alvarez. 2010. Predicting election outcomes from positive and negative trait assessments of candidate images. Political Psychology 31, 1 (2010), 41-58.

[23] Shannon C McGregor. 2018. Personalization, social media, and voting: Effects of candidate self-personalization on vote intention. new media \& society 20,3 (2018), 1139-1160.

[24] Shannon C McGregor, Regina G Lawrence, and Arielle Cardona. 2017. Personalization, gender, and social media: gubernatorial candidates' social media strategies. Information, Communication \& Society 20, 2 (2017), 264-283.
[25] Cecilia Hyunjung Mo. 2015. The consequences of explicit and implicit gender attitudes and candidate quality in the calculations of voters. Political behavior 37, 2 (2015), 357-395.

[26] Shirley Miller Rosenwasser and Norma G Dean. 1989. Gender role and political office: Effects of perceived masculinity/femininity of candidate and political office. Psychology of Women Quarterly 13, 1 (1989), 77-85.

[27] Erik Tjong Kim Sang and Johan Bos. 2012. Predicting the 2011 dutch senate election results with twitter. In Proceedings of the workshop on semantic analysis in social media. 53-60.

[28] Pamela J Shoemaker and Timothy Vos. 2009. Gatekeeping theory. Routledge.

[29] Marko Skoric, Nathaniel Poor, Palakorn Achananuparp, Ee-Peng Lim, and Jing Jiang. 2012. Tweets and votes: A study of the 2011 singapore general election. In 2012 45th Hawaii international conference on system sciences. IEEE, 2583-2591.

[30] Zachary C Steinert-Threlkeld. 2019. The Future of Event Data Is Images. Sociological Methodology 49, 1 (2019), 68-75.

[31] Andranik Tumasjan, Timm O Sprenger, Philipp G Sandner, and Isabell M Welpe. 2010. Predicting elections with twitter: What 140 characters reveal about political sentiment. In Fourth international AAAI conference on weblogs and social media.

[32] Yu Wang, Yang Feng, Jiebo Luo, and Xiyang Zhang. 2016. Pricing the woman card: Gender politics between Hillary Clinton and Donald Trump. In 2016 IEEE International Conference on Big Data (Big Data). IEEE, 2541-2544.

[33] Yu Wang, Yuncheng Li, and Jiebo Luo. 2016. Deciphering the 2016 US Presidential campaign in the Twitter sphere: A comparison of the Trumpists and Clintonists. In Tenth International AAAI Conference on Web and Social Media.

[34] Donghyeon Won, Zachary C Steinert-Threlkeld, and Jungseock Joo. 2017. Protest activity detection and perceived violence estimation from social media images. In Proceedings of the 25th ACM international conference on Multimedia. ACM, 786-794.

[35] Nan Xi, Di Ma, Marcus Liou, Zachary C Steinert-Threlkeld, Jason Anastasopoulos, and Jungseock Joo. 2019. Understanding the Political Ideology of Legislators from Social Media Images. arXiv preprint arXiv:1907.09594 (2019).

[36] Quanzeng You, Liangliang Cao, Yang Cong, Xianchao Zhang, and Jiebo Luo. 2015. A multifaceted approach to social multimedia-based prediction of elections. IEEE Transactions on Multimedia 17, 12 (2015), 2271-2280.

[37] Han Zhang and Jennifer Pan. 2019. CASM: A Deep-Learning Approach for Identifying Collective Action Events with Text and Image Data from Social Media. Sociological Methodology 49, 1 (2019), 1-57.

[38] Junhuan Zhu, Jiebo Luo, Quanzeng You, and John R Smith. 2013. Towards understanding the effectiveness of election related images in social media. In 2013 IEEE 13th International Conference on Data Mining Workshops. IEEE, 421-425. 